\title{
Impulse Based Scheme for Crystal-less ULP Radios
}

\author{
Fabio Sebastiano*, Salvatore Drago*, Lucien Breems*, Domine Leenaerts*, Kofi Makinwa ${ }^{\dagger}$ and Bram Nauta ${ }^{\ddagger}$ \\ ${ }^{*}$ NXP Semiconductors, Eindhoven, The Netherlands, Email: \{fabio.sebastiano, salvatore.drago $\} @ n x p . c o m$ \\ $\dagger$ Electronic Instrumentation Laboratory, Delft University of Technology, Delft, The Netherlands \\ $\ddagger$ IC Design Group, CTIT Research Institute, University of Twente, Enschede, The Netherlands
}

\begin{abstract}
This work describes a method of implementing a fully-integrated Ultra-Low Power (ULP) radio for Wireless Sensor Networks (WSN). This is achieved using a specific Medium Access Control (MAC) protocol, employing a dutycycled wake-up radio and a crystal-less clock generator, and an ad-hoc modulation scheme (Impulse Radio) with a bandwidth of 17.7 MHz in the $2.4 \mathrm{GHz}$ - ISM band. The total average power consumption is expected to be less than $100 \mu \mathrm{W}$.
\end{abstract}

\section{INTRODUCTION}

Wireless Sensor Networks (WSN) require transceivers that are small, cheap and power efficient. The largest fraction of the energy in each node of a WSN is spent in idle listening to the channel [1]. Previous solutions focus on the use of a reactive radio [2] or on synchronous networks [3]. In the latter, nodes are equipped with a high accuracy clock, which can hardly be integrated. In the reactive radio approach, in addition to the main radio, i.e. the radio used for communication, nodes are equipped with a wake-up radio with very low power consumption, as very simple architectures employing high-Q off-chip RF filters [4], [5]. At the current state-of-the-art, no radio with lower power consumption than $100 \mu \mathrm{W}$ with acceptable sensitivity has been proposed.

The issue of integration is also present in the main radio. An accurate frequency reference is needed to respect spectrum regulations and to tune the receiver to the incoming signal. In previous solutions, an accurate RF frequency reference is obtained using at least one external component, i.e. quartz crystals, BAWs or MEMS.

This work deals with a low power implementation and complete integration of a WSN: duty-cycling the wake-up radio and employing impulse based modulation, a crystal-less system is obtained. The MAC protocol is described in section II; the physical level and its influence on both the main receiver and the wake-up radio are described in detail respectively in section III and IV. Conclusions are drawn in section V.

\section{MAC PROTOCOL: DUTY-CYCLED WAKE-UP RADiO}

In the proposed scheme, the receiver, while residing in a reduced power mode (sleep mode), is able to decide when to turn itself on to listen for communications (listening mode), and when communications are present, to prompt a full power-up of the device (communication mode). This can be implemented by the architecture in Fig. 1(a), comprising a wake-up radio responsible to monitor the channel waiting for data packets and a main radio to communicate. The wake-up radio is duty-cycled, i.e. put in listening mode on a scheduled

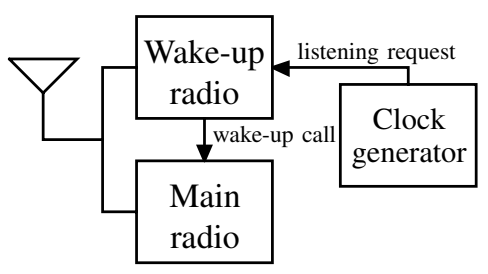

(a)

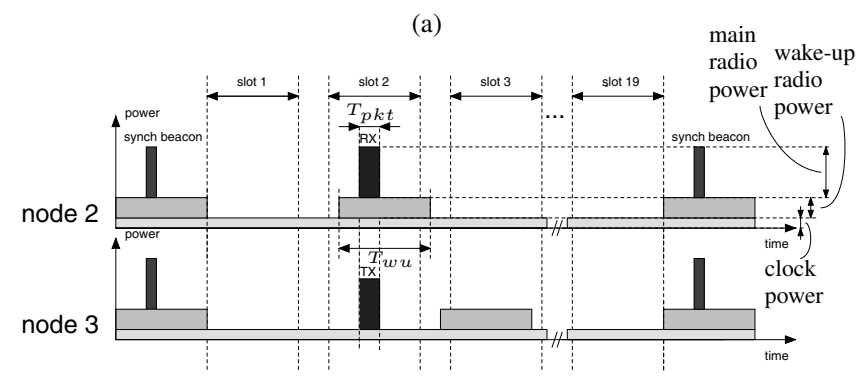

(b)

Fig. 1. (a) System architecture and (b) MAC protocol with energy breakdown.

basis by a clock generator to save energy when monitoring is not required. When a data packet is present, the wake-up radio prompts a wake-up call for the main radio. As shown in Fig. 1(b), time is divided into fixed slots, e.g. Slot 1, Slot $2, \ldots$, which form the basis of the Time Division Multiple Access (TDMA) protocol. Accordingly, on each time slot only the wake-up radio of a particular node is allowed to monitor the channel. For example, time Slot 2 can be allocated to Node 2, time Slot 3 to Node 3 and so on. Any node can transmit a packet in any slot, depending on the intended recipient node of the data packet. In the figure, Node 3 sends a packet to Node 2 in time Slot 2. Specialized timeslots, used for the time synchronization of the whole network, are labelled in Fig. 1(b) as synchronization beacons. All nodes listen to the synchronization beacons and reset their internal clock at the reception of the beacon. A particular node, called master, periodically sends the synchronization beacon.

The duration of the listening timeslots should be long enough to account for timing errors between the clocks of receiver and transmitter to ensure that a packet is transmitted when the recipient is in listening mode. Since the clocks are reset at the synchronization beacon time instances, timing errors in receivers and transmitters are accumulated from the last beacon and will depend on clock accuracy.

Duty-cycling the wake-up radio together with the TDMA 
protocol reduces the energy spent listening to the channel, and relaxes constraints on power consumption. As such, the allowed skew between clocks is relaxed, and fully integrated clocks, with moderate accuracy of $5000 \mathrm{ppm}$ or less, can be used. Moreover, since the power budget for the wake-up radio is increased by duty-cycling, its sensitivity can be increased without off-chip filters. As an example, if the clock accuracy is $1 \%$, the synchronization beacon period and the duration of reception slots can be $10 \mathrm{~s}$ and $400 \mathrm{~ms}$, respectively. As the synchronization beacons occupy a time slot of equal duration to the data slots and assuming that a node has a timeslot at least once per minute, the wake-up radio has a duty-cycle of $4.67 \%$ and the fraction of total average power due to a $500 \mu \mathrm{W}$ wake-up radio is only $23.3 \mu \mathrm{W}$. Note that, also considering $2 \mathrm{~mW}$ power consumption during packet transmission and reception, the fraction of total average power is only $0.53 \mu \mathrm{W}$ with a packet length of 200 bits, a date rate of $100 \mathrm{kbps}$ and an average packet rate of $1 \mathrm{pkt} / \mathrm{min}$, resulting in a dutycycle of $0.027 \%$. With a reasonable power consumption for the clock of $50 \mu \mathrm{W}$ [6], the total average power consumption for this approach is $73.9 \mu \mathrm{W}$, which is below typical energy constraints for low power consumption WSNs.

\section{IMPULSE RADIO MODULATION}

\section{A. Modulation and band allocation}

RF modulation must be chosen to relax as much as possible the frequency accuracy requirements. For narrowband modulations (such as OOK, FSK or QAM), the specifications on frequency accuracy is very strict, since the allowed frequency error required at the receiver is directly proportional to the bandwidth of the RF signal. A possible solution can be the adoption of an Ultra Wide Band (UWB) system employing Impulse Radio (IR) modulation scheme [7]; the RF signal occupies a bandwidth of hundreds of $\mathrm{MHz}$ to comply with the radio regulations and a fully integrated reference for this application can be easily built. However, it will be difficult to meet the power requirements at the receiver due to the inherent wideband nature of UWB receiver. A better solution is the use of an Impulse Radio signal with a bandwidth smaller than that required in UWB systems but large enough to relax frequency accuracy constraints for full integration.

The preferred band is the $2.4 \mathrm{GHz}$ ISM-band, which allows occupation of tens of $\mathrm{MHz}$ at a relatively high frequency which enables the integration of required passive components (as inductors) on chip. Fig. 2 shows the simplified time representation of the adopted signal and its spectrum. An RF carrier is modulated by a pulse waveform with period $T_{f}$ and duty cycle $T_{p} / T_{f}$. The pulses are shaped as square waves with duration $T_{p}$ and pulse repetition frequency $P R F=1 / T_{f}$. Each bit is represented by a sequence of $n=P R F / D R$ successive pulses, where $D R$ is the data rate, implementing the well-known repetition code. The bits are modulated using Pulse Position Modulation (PPM). In each frame, i.e. in each slot of duration $T_{f}$ the pulse can be transmitted with different delays: the pulse can be positioned with zero delay (bit 0 ) or with delay $T_{p p m}$ (bit 1), in case of binary modulation;

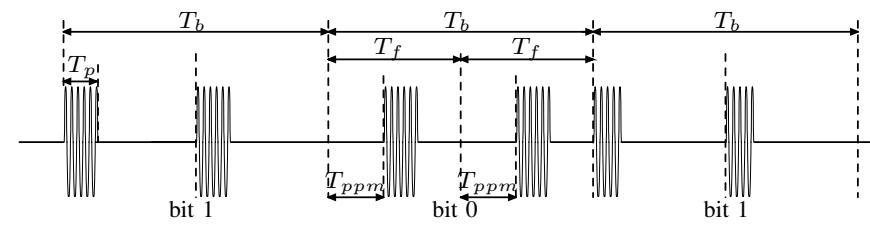

(a)

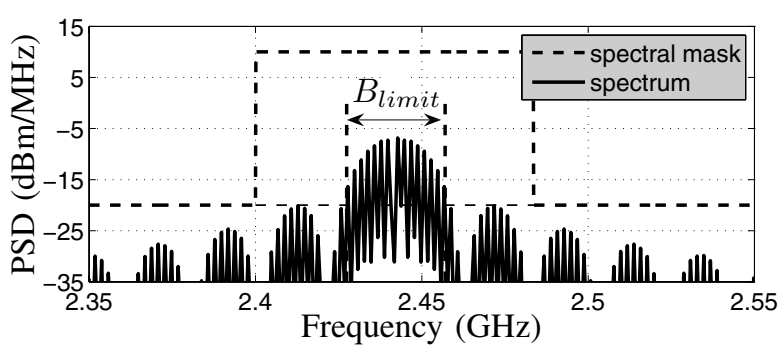

(b)

Fig. 2. Example of IR signal (a); spectrum with parameters of Table I (b).

more delays can be added to employ an M-ary modulation. In the following, constraints affecting the modulation parameters choice are listed in order to find an optimal set of parameters.

\section{B. Transmitter limits}

With an average transmitted power ${ }^{1} P_{\text {avg }}=1 \mathrm{~mW}$, the European ETSI $2.4 \mathrm{GHz}$ ISM band requirements are met if the transmission frequency resides in the interval $\left[f_{0}-\Delta f_{T X}, f_{0}+\Delta f_{T X}\right]$, where $f_{0}=2.44175 \mathrm{GHz}$ is the nominal transmitting frequency at the center of ISM band and

$$
\Delta f_{T X}=41.75 \mathrm{MHz}-\frac{B_{\text {limit }}\left(T_{p}\right)}{2}
$$

where $B_{\text {limit }}$ is the width of the spectrum of Fig. 2(b) at $-20 \mathrm{dBm} / \mathrm{MHz}$ and it is numerically computed.

The complexity of transmitter circuitry depends on the signal Crest Factor, defined as $C F \triangleq \frac{P_{p e a k}}{P_{a v g}}=\frac{T_{f}}{T_{p}}$ where $P_{\text {peak }}$ is the peak power. In order not to put excessive requirements on the transmitter, the crest factor has to be chosen less than $C F_{T X, \max }=10$. The following condition must hold:

$$
C F=\frac{T_{f}}{T_{p}} \leq \min \left\{C F_{T X, \text { max }}, \frac{P_{\text {peak }, \text { limit }}}{P_{\text {avg }}}\right\}=10
$$

where $P_{\text {peak,limit }}$ is fixed by the regulations.

\section{Receiver limits}

To simplify the architecture, we assume the use of the repetition coding and of a noncoherent receiver. Both hard and soft detection algorithms can be used for decoding but it can be proven that soft decoding is less robust to frequency mismatches between transmitter and receiver. Thus, hard detection is employed, according to which the received bit is

\footnotetext{
${ }^{1}$ The power of the transmitted signal is limited by the power budget of the node; considering the power analysis carried in section II and the expected efficiency of the transmitter, $P_{a v g}=1 \mathrm{~mW}$ is a reasonable assumption. Note that the spectral mask requirements are still met if less power than $1 \mathrm{~mW}$ is emitted and the assumed value is a practical upper bound.
} 


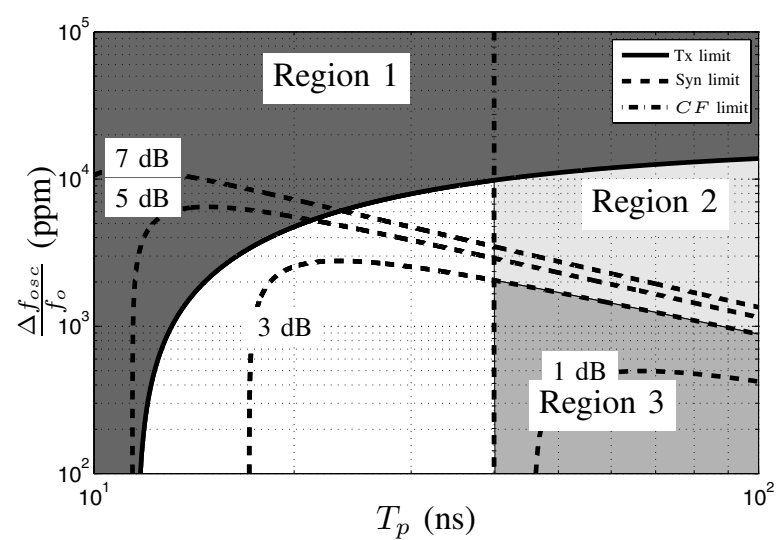

Fig. 3. Plot for the choice of $T_{p}$; different constraint are shown: Tx limit $\left(\frac{\Delta f_{o s c}}{f_{0}} \leq \frac{\Delta f_{T X} X}{f_{0}}\right)$, Syn limit $\left(\frac{\Delta f_{o s c}}{f_{0}} \leq \frac{\Delta f_{s y n}}{2 f_{0}}\right)$ for various $I L_{s y n}$ (shown
on the curves) and $C F$ limit (5).

decided with the majority criterion on the $n$ received pulses. For a fixed $P_{a v g}$ and $D R$, the implementation loss related to the decoding algorithm increases with the number of pulses per bit $n$; consequently the following practical limit is posed:

$$
n=\frac{T_{b}}{T_{f}} \leq 25
$$

which corresponds to an implementation loss of $5.1 \mathrm{~dB}$ in receiver sensitivity for a Bit Error Rate (BER) of $10^{-3}$.

\section{Synchronization requirements}

The receiver synchronizes in time and frequency to the incoming signal using a preamble. In case a not perfect recovery is performed, an implementation loss must be taken into account for the sensitivity ${ }^{2}$ :

$$
I L_{\text {syn }}=\frac{2\left(\pi \Delta f_{\text {syn }} T_{p}\right)^{2}}{1-\cos \left[\left(2 \pi \Delta f_{\text {syn }}\left(T_{p}-\left|\Delta t_{\text {syn }}\right|\right)\right]\right.}
$$

where $\Delta f_{\text {syn }}$ and $\Delta t_{\text {syn }}$ are respectively the error in frequency and timing between the actual value and the estimated one at the receiver.

\section{E. Optimization}

The choice of $T_{p}$ depends on $\Delta f_{\text {osc }}$, defined as the error of the local oscillator frequency with respect to the nominal frequency. All previous constraints are plotted in Fig. 3: $\Delta f_{\text {osc }} \leq \Delta f_{T X}$ (with reference to (1)) in solid line; in dasheddotted line the $C F$ limit, i.e.

$$
\frac{T_{f}}{T_{p}} \cdot \frac{T_{b}}{T_{f}} \leq 10 \cdot 25 \Rightarrow T_{p} \geq \frac{T_{b}}{250}=40 \mathrm{~ns}
$$

derived using (2) and (3), where $T_{b}$ is assumed to be $10 \mu \mathrm{s}$ $(D R=100 \mathrm{kbps})$ in our application; the synchronization limit in dashed line. Synchronization limit is obtained from (4) for different values of $I L_{s y n}$ : the condition ${ }^{3} \Delta f_{\text {osc }} \leq \frac{\Delta f_{\text {syn }}}{2}$ is

\footnotetext{
${ }^{2}$ The formula is simply obtained calculating the output of a matched filter receiver with timing and frequency errors.

${ }^{3}$ The factor 2 derives from the presence of frequency errors both in transmitter and receiver.
}

TABLE I

MODULATION PARAMETERS

\begin{tabular}{|l|l|l|l|l|l|}
\hline \multicolumn{2}{|l|}{ Data rate $(D R)$} & $100 \mathrm{kbps}$ & \multicolumn{2}{l|}{$P_{\text {avg }}$} & $1 \mathrm{~mW}$ \\
\hline \multicolumn{2}{|l|}{$-3 \mathrm{~dB}$ bandwidth } & $17.7 \mathrm{MHz}$ & \multicolumn{1}{l|}{$C F$} & 9.52 \\
\hline IR parameters & $T_{f}$ & $476 \mathrm{~ns}$ & Synchro- & $I L_{\text {syn }}$ & $3 \mathrm{~dB}$ \\
& $T_{p p m}$ & $238 \mathrm{~ns}$ & nization & $\Delta f_{\text {syn }}$ & $8.4 \mathrm{MHz}$ \\
& $T_{p}$ & $50 \mathrm{~ns}$ & parameters & $\Delta t_{\text {syn }}$ & $5 \mathrm{~ns}$ \\
\hline
\end{tabular}

plotted with the timing error of the synchronization algorithm fixed to $\left|\Delta t_{\text {syn }}\right|=5 \mathrm{~ns}$. If the accuracy of the oscillator is enough for a given $I L_{\text {syn }}$, no frequency estimation must be performed at the receiver. Since it is possible to trade off timing error $\left(\Delta t_{s y n}\right)$ for frequency error $\left(\Delta f_{\text {syn }}\right)$ in (4), a small timing error ( $5 \mathrm{~ns}$ ) has been chosen. This is advantageous in terms of hardware as it is much more difficult to tune the frequency of the receiver than the timing.

From Fig. 3 a good choice for $T_{p}$ can be found according to frequency accuracy considerations. Referring to an $I L_{\text {syn }}$ of $3 \mathrm{~dB}$, it is possible to distinguish different regions. In region 1 , i.e. all the points above the transmission limit, the transmitter will not respect the spectral mask. Points in region 2 respect the transmission limit but some frequency synchronization at the receiver is needed because the frequency accuracy is not enough. Region 3, i.e. the points under the transmission limit and under the curve of $I L_{\text {syn }}=3 \mathrm{~dB}$, contains points for which no frequency synchronization is required at the receiver because the frequency accuracy provided by the local oscillator is enough to mantain $I L_{\text {syn }}$ below $3 \mathrm{~dB}$. The optimal point is strictly related to the available oscillator frequency accuracy. For example with an accuracy of $0.2 \%$, it is possible to find out that a choice of $T_{p}=40 \mathrm{~ns}$ allows to enter the optimal region 3. If, however, a good frequency reference like a crystal oscillator is not available, it is very challenging to reach an accuracy below $0.5 \%$. With such a number, for any value of $T_{p}$, frequency synchronization must be performed in order to keep an implementation loss due to the synchronization system less than $3 \mathrm{~dB}$. A choice of $T_{p}=50 \mathrm{~ns}$ has been taken, giving an allowed absolute timing and frequency error equal to $5 \mathrm{~ns}$ and $8.44 \mathrm{MHz}$ respectively.

The frame period $T_{f}$ is chosen accordingly to different requirements: (2), (3) and $P R F<2.5 \mathrm{Mpps}$ (to avoid a very fast baseband). Thus, $T_{f}=476 \mathrm{~ns}$ is employed and consequently, $P R F=2.1 \mathrm{Mpps}, n=21$ and $C F=9.52$. The resulting modulation parameters are reported in Table I.

\section{WAKE-UP RADIO}

\section{A. Architecture and performances}

IR modulation is beneficious also for the wake-up functionality. Since the peak power of the pulses is higher than the average power of the received signal, the wanted signal can be discriminated from noise using a nonlinear receiver, making narrowband filtering unnecessary. 
A block diagram of the chosen architecture and the related waveforms are shown in Fig. 4. When the signal of Fig. 2(a) is present at the input and the noise is low enough, the system detects the envelope of the signal and recognizes pulses longer than a certain pulse length threshold. The digital output (Hit) is high when a pulse is detected; the system is reset on each frame period $\left(T_{f}\right)$ and the value of the digital output for each frame is stored. After the observation of $m$ frames periods, the number of hits, i.e. the number of times a pulse was detected in the $m$ frames, is counted. If $m$ hits have been detected, a wake-up call for the main radio is issued; otherwise the count is reset and the procedure start again.

Matlab $^{\mathrm{TM}}$ simulations ${ }^{4}$ were performed to characterize the architecture of Fig. 4(a). It can be completely characterized plotting the Signal Hit Probability, i.e. the probability to have a hit for a frame where a pulse is present, and the Noise Hit Probability, i.e. the probability to have a hit for a frame with no pulse but only noise. In Fig. 5 results are shown as a function of Signal-to-Noise Ratio (SNR) and for different pulse length threshold. As the pulse length threshold is increased, Noise Hit Probability is lowered, but Signal Hit Probability is worsened. Consequently, a pulse length threshold of $45 \mathrm{~ns}$ and a minimum SNR of $-3 \mathrm{~dB}$ are adopted.

From Noise Hit Probability and Signal Hit Probability for this case, the missed detection probability and the false alarm probability ${ }^{5}$ can be computed as a function of the number of frames $m$ used for signal detection. For $m=10$, i.e. for less than one bit, the probability of false alarm is less than $10^{-10}$ and it can be neglected. In the same condition, the probability of missed detection is also smaller than that ${ }^{6}$.

\section{B. Notes on implementation}

With no narrow band filtering at the antenna, the noise bandwidth of the system is assumed larger than $100 \mathrm{MHz}$. For $100 \mathrm{MHz}$ noise bandwidth and $-84 \mathrm{dBm}$ sensitivity, an input referred $\mathrm{SNR}=-3 \mathrm{~dB}$ is equivalent to a total radio noise figure of $13 \mathrm{~dB}$. Assuming a minimum allowable signal of $60 \mathrm{mVpp}$ amplitude at the envelope detector input, a voltage gain of $60 \mathrm{~dB}$ is required before the envelope detector, with an antenna impedance of $50 \Omega$ and the parameters of Table I. This seems possible with $500 \mu \mathrm{W}$ power consumption [8].

\section{CONClusion}

Low power consumption and fully integration of nodes in a WSN can be achieved if ad-hoc MAC and modulation schemes are employed. Duty-cycling the wake-up radio, energy spent

\footnotetext{
${ }^{4}$ Simulation specifications: $A W G N$ channel; $S N R$ is the signal-to-noise ratio at the input of the comparator; a signal of $-84.2 \mathrm{dBm}$ at $2.44 \mathrm{GHz}$ and $C F=10$ was employed; the threshold of the comparator was set to $-75.2 \mathrm{dBm}$; the envelope detector had an exponential discharge behaviour with time constant of $10 \mathrm{~ns}$.

${ }^{5}$ The missed detection probability is the probability to miss an incoming packet; the false alarm probability is the probability to issue a wake-up call due only to noise.

${ }^{6}$ Interfers can trigger the wake-up radio and produce false wake-ups. Though the analysis of interferers effect is out of the scope of this paper, it can be noted that rejection of interferers is increased employing an higher threshold (Fig. 4) and multi-hop routing to reduce inter-node distance.
}

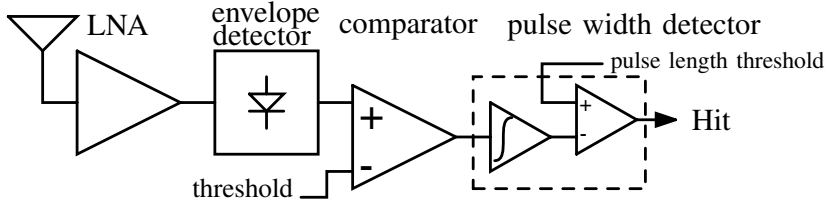

(a)

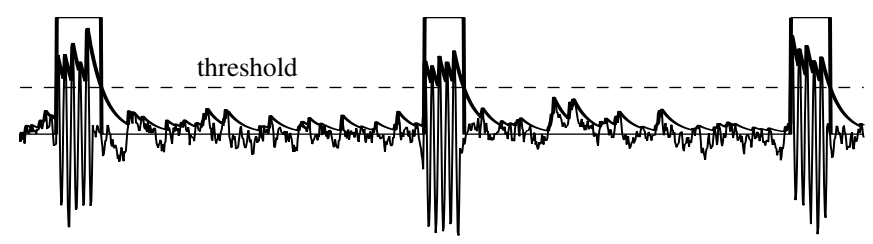

(b)

Fig. 4. Wake-up radio architecture (a) and example related waveforms (b): the outputs of LNA, envelope detector and comparator are shown.
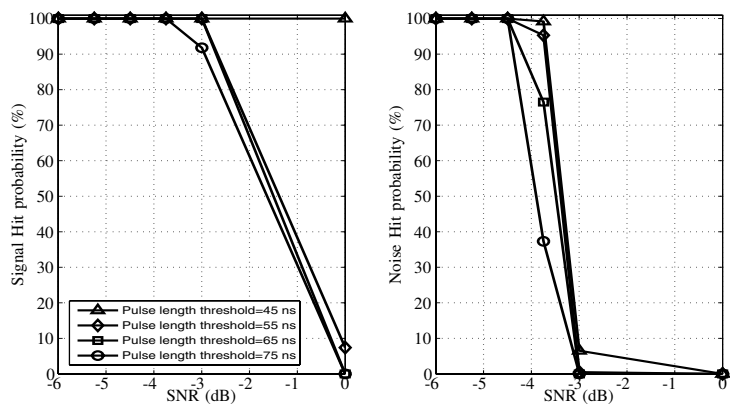

Fig. 5. Signal Hit Probability and Noise Hit Probability versus SNR for different pulse length threshold.

in idle monitoring can be reduced, while maintaining the fully integration on chip. By using an ad-hoc modulation scheme the requirements on frequency accuracy can be relaxed enabling the use of crystal-less oscillators and the design of simple wake-up radios without external components.

\section{REFERENCES}

[1] J. Ammer, et al., "Ultra low-power integrated wireless nodes for sensor and actuator networks," in Ambient Intelligenence, W. Weber, J. M. Rabaey, and E. Aarts, Eds. Springer, 2005.

[2] J. M. Rabaey, et al., "PicoRadios for wireless sensor networks: the next challenge in ultra-low power design," in ISSCC, Dig. of Tech. Papers, vol. 1, Feb. 2002, pp. $200-201$

[3] C. C. Enz, A. El-Hoiydi, J.-D. Decotignie, and V. Peiris, "Wisenet: an ultralow-power wireless sensor network solution," Computer, Aug. 2004.

[4] D. C. Daly and A. P. Chandrakasan, "An energy efficient OOK ook transceivers for wireless sensor networks," in IEEE Radio Frequency Integrated Circuits (RFIC) Symposium, June 2006.

[5] B. W. Otis, Y. H. Chee, R. Lu, N. M. Pletcher, and J. M. Rabaey, “An ultra-low power MEMS-based two-channel transceiver for wireless sensor networks," in VLSI Circuits, Dig. of Tech. Papers, June 2004.

[6] G. D. Vita, F. Marraccini, and G. Iannaccone, "Low-voltage low-power CMOS oscillator with low temperature and process sensitivity," in Proc. ISCAS 2007, May 2007, pp. 2152-2155.

[7] J. Ryckaert, et al., "A 16mA UWB 3-to-5GHz 20Mpulses/s quadrature analog correlation receiver in $0.18 \mu \mathrm{m}$ CMOS," in ISSCC Digest of Technical Papers., Feb. 2006, pp. $114-115$.

[8] D. C. Daly and A. P. Chandrakasan, "An energy-efficient OOK transceiver for wireless sensor networks," IEEE J. Solid-State Circuits, no. 5, pp. 1003 - 1011, May 2007. 